\title{
PERCEPÇÃO DOS ESTUDANTES DE MEDICINA ACERCA DA RESIDÊNCIA EM MEDICINA DE FAMÍLIA E COMUNIDADE
}

\section{PERCEPTIONS OF MEDICAL STUDENTS ON \\ THE RESIDENCE IN FAMILY AND COMMUNITY MEDICINE}

\author{
Aline Stela Tinoco ${ }^{1}$ \\ InaJara CARLa Oliveira ${ }^{2}$ \\ Luiz Roberto Agea Cutolo ${ }^{3}$ \\ Marcos Aurélio Maeyama ${ }^{4}$
}

1 Acadêmica do $10^{\circ}$ período do Curso de Medicina pela Universidade do Vale do Itajaí (UNIVALI).E-mail: alinetinoco@, outlook.com.

2 Mestre em Saúde e Gestão do Trabalho - UNIVALI. Especialista em Saúde da Família Fonoaudióloga - UNIVALI. E-mail: ina_carla@hotmail.com.

3 Doutor em Educação, Pediatra, professor da Universidade Federal de Santa Catarina (UFSC). E-mail: lu.cutolo@gmail.com.

4 Doutor em Saúde Coletiva Universidade Federal de Santa Catarina (UFSC). Odontólogo, professor da UNIVALI. E-mail: marcosmaeyama@ig.com.br.
RESUMO: Introdução: Ainda há poucos dados na literatura que indiquem as percepções dos estudantes pela residência em Medicina de Família e Comunidade (MFC) no contexto de escolha da especialidade médica. Objetivo: Identificar o perfil dos estudantes formandos no Curso de Medicina da Universidade do Vale do Itajaí (UNIVALI) no ano de 2016, e assim analisar suas percepções sobre a Medicina da Família e Comunidade no contexto de escolha da especialidade médica. Metodologia: Tratase de uma pesquisa com abordagem analítico-descritiva, tendo como técnica de coleta de dados a aplicação de um questionário previamente elaborado pelos pesquisadores, contendo perguntas objetivas. Este estudo contou com a participação de 21 acadêmicos formandos no Curso de Medicina da Região do Vale do Itajaí do primeiro semestre do ano de 2016. Resultados: Os alunos que responderam à pesquisa apresentaram média de idade de 26 anos. Quanto ao gênero, a pesquisa identificou o predomínio do sexo feminino, totalizando $57,1 \%$ (12 sujeitos) da amostra. É possível inferir que os fatores mais levados em conta na opinião dos acadêmicos na escolha por MFC foram em primeiro lugar a "carga horária relativamente baixa com horários definidos", juntamente com "olhar médico ampliado, além do biomédico", ambos correspondendo a $57,1 \%$, seguido da "oportunidade de trabalho em qualquer região", 52,4\% e, por fim, a "atuação ampla em diversas disciplinas médicas", 47,6\%. Conclusão: Mesmo frente ao movimento de mudança desta realidade proposta pelo governo, observa-se que estas ainda são incipientes, e assim continuarão se não propuserem uma maior consolidação e incentivo financeiro para valorização profissional desta área.

PALAVRAS-CHAVE: Medicina de Família e Comunidade; Medicina Comunitária; Residência médica; Especialidade médica.

ABSTRACT: There is still little data in the literature that indicates students' perceptions on the Residence in Family and Community Medicine (FCM) in the 
context of choosing a medical specialty. Objective: To identify the profile of students taking a medical degree at the University of Vale do Itajaí (UNIVALI) in 2016, and to analyze their perceptions about FCM in the context of choosing a medical specialty. Methodology: This research used a quantitative approach. For the data collection, it applied a questionnaire, previously prepared by the researchers, with open-ended questions. This study included 21 students taking the Course in Medicine in the Vale do Itajaí Region in the first semester of 2016. Results: The average age of the students who answered the questionnaire was 26 years. In terms of sex, a predominance of females was identified (57.1\%, or 12 subjects) in the sample. It can be inferred that the factors most taken into account in the students' opinions in the decision to study FCM were: firstly, the "relatively low hours with set timetables" together with "extended medical care, besides biomedical care", both corresponding to $57.1 \%$, followed by "job opportunities in any region", with 52.4\%, and finally the "broad performance in various medical disciplines", with $47.6 \%$. Even faced with the movement of changes of this reality proposed by the government, it is observed that these are still incipient, and they will remain so unless they propose a greater consolidation and financial incentive for professional appreciation of this area.

KEYWORDS: Family practice; Community medicine; Residency; Medical specialty.

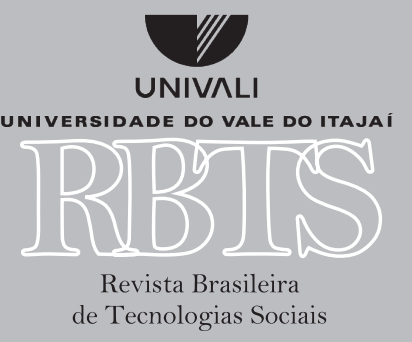

Revista Brasileira de Tecnologias Sociai 


\section{INTRODUÇÃO}

A Medicina de Família e Comunidade (MFC) é a especialidade apta a coordenar o cuidado da saúde das pessoas, de forma longitudinal, integral, considerando seu contexto individual, familiar e comunitário (ANDERSON; GUSSO; FILHO, 2005). Foi considerada para Rodrigues e Anderson (2011) como a especialidade médica que mais se adequou aos objetivos propostos pela Organização Mundial de Saúde ao consagrar os programas de Atenção Primária à Saúde como prioridade das nações participantes na área. Tem potencial transformador tanto da prática médica quanto na formação de recursos humanos (ANDERSON; DEMARZO; RODRIGUES, 2007).

Rodrigues e Anderson (2011), após análise de estudos passados, mostraram que países que organizam seus sistemas de saúde dando maior atenção em cuidados primários atendem com mais eficiência as necessidades de saúde das pessoas e de toda a comunidade. O que se observa na prática é que os países que conseguiram efetivamente implantar este modelo obtiveram sucesso, apresentando populações mais saudáveis e com redução dos efeitos das desigualdades sociais. Cerca de $85 \%$ dos problemas de saúde relativos a uma população específica, sem diferenciação de sexo ou faixa etária, podem ser resolvidos por uma equipe de estratégia saúde da família, na qual se inclui médico de família (ANDERSON; GUSSO; FILHO, 2005).

Para Lopes e Ribeiro (2015), a resolução dos problemas de saúde pressupõe o atendimento das singularidades a partir de uma atenção centrada no indivíduo e estabelecendo relações de determinação social. Ainda segundo estes autores, este tipo de ação é considerado fundamental para um bom desempenho de qualquer profissional da área da saúde, mas ao médico de família e comunidade ela é imprescindível.

No Brasil, apesar de a especialidade em MFC existir desde 1976, foi só em 1994 que ela começou a ganhar destaque, com a criação do Programa Saúde da Família (PSF), atual Estratégia Saúde da Família (NETO; LIRA; MIRANDA, 2009). Mesmo considerando prematuro levantar hipóteses para o pouco interesse dos profissionais pela área, esta pode provir de uma dificuldade dos estudantes de Medicina em eleger qual a verdadeira necessidade da saúde hoje, devido ao fascínio que o complexo médico industrial exerce sobre o imaginário de potenciais usuários e de profissionais (LUCCHESE 2003). Contudo, são necessários estudos que visem descrever o problema em sua complexidade, para que se possam estabelecer estratégias no âmbito das políticas de saúde e da educação médica eficazes e efetivas para enfrentá-lo (NETO; LIRA; MIRANDA, 2009).

Segundo Campos (2005), inúmeras doenças não podem ser totalmente compreendidas sem serem vistas em seu contexto pessoal, familiar e comunitário. Assim, é necessário um olhar ampliado para as necessidades dos indivíduos e das populações, conforme Anderson e Rodrigues (2011) apontam a seguir:

[...] as necessidades de cuidados primários que as pessoas experimentam ao longo de suas vidas exigem mais do que o emprego de procedimentos focais, transitórios ou pontuais, voltados para o controle de instabilidades fisiopatológicas críticas. São necessidades de promoção da saúde, prevenção tanto das doenças evitáveis quanto da instalação precoce das enfermidades crônicas e das suas complicações que levam à invalidez ou morte prematuras. (p.1-2)

Desta forma, ao considerar o desenvolvimento de ações para além da prevenção de doenças, e com grande fomento para promoção da saúde, demanda-se uma concepção ampliada 
sobre o processo saúde e doença, exercício este necessário desde a graduação, a fim de estabelecer um novo modelo de profissional médico. Contudo, a formação deste novo profissional, capaz de resolver com competência 85\% dos problemas inerentes à Atenção Básica, requer uma verdadeira mudança de paradigma educacional no ambiente acadêmico. Tem-se um variado universo de profissionais atuando no campo da medicina generalista, sendo que poucos estão lá por verdadeira opção. Muitos deles simplesmente não conseguiram especializar-se e por fim outros que têm que preencher um vazio que é, na prática, mais necessitado de mão de obra do que de ação política (JANAUDIS et al., 2007).

Diante do apresentado, a presente pesquisa tem como objetivo identificar quais os fatores que interferem e influenciam a escolha do recém-formado pela MFC, quando este opta pela mesma. Espera-se com isso promover possíveis mudanças na matriz curricular do Curso de Medicina, as quais tornem os alunos mais motivados, se este for o caso, ou quem sabe, buscar formas de repensar sobre esta especialidade no contexto brasileiro.

\section{CONSIDERAÇÕES METODOLÓGICAS E ÉTICAS}

Esta foi uma pesquisa de campo realizada mediante e após a aprovação de prerrogativas éticas exigidas na resolução CNS 466/12, bem como aprovação do Comitê de Ética em Pesquisa (CEP) sob o parecer $n^{\circ} 1.371 .233$.

Este estudo faz parte de um projeto uma pesquisa de caráter analítico-descritivo, que teve como objetivo compreender as percepções dos estudantes formandos do Curso de Medicina no ano de 2016 acerca da residência em Medicina de Família e Comunidade (MFC) no contexto de escolha da especialidade médica.

Para a realização da pesquisa, foram convidados os estudantes do Curso de Medicina da Região do Vale do Itajaí que estavam cursando o último semestre do mesmo (no decorrer do primeiro semestre de 2016), devido ao fato destes já terem tido a experiência com a MFC durante a graduação, bem como com as demais especialidades médicas, compondo assim uma amostra intencional. No universo de 33 alunos formandos, a população estudada foi constituída por uma casuística de 21 estudantes, correspondendo a 63,63\% da população alvo.

Como critérios de inclusão, há: 1) Estudantes formandos do Curso de Medicina do primeiro semestre de 2016;2) Estudantes que tiveram disponibilidade de tempo para responder o questionário; 3) Aceitaram participar da pesquisa e assinaram o termo de consentimento livre e esclarecido.

A coleta de dados deu-se por meio da aplicação de um questionário previamente elaborado pelas pesquisadoras, contendo perguntas objetivas e abertas a partir de um modelo de Figueiredo modificado (2013). Vale destacar que a aplicação do questionário ocorreu de maneira agendada, durante os intervalos de estágios dos graduandos, em uma sala reservada, após o convite para participação da pesquisa via correio eletrônico. Os dados coletados a partir da aplicação do questionário apresentavam a possibilidade de assinalar mais de um item em uma mesma pergunta objetiva. Estas perguntas visaram à identificação do perfil dos formandos com relação aos aspectos de idade e gênero. No que se refere à opção pela especialidade médica, os alunos foram indagados sobre: intenção em realizar residência em MFC, nível de satisfação com a disciplina durante a graduação, motivos pelos quais se sentiam atraídos e não se sentiam atraídos pela residência em 
MFC, qual a sua primeira opção de especialidade médica. Na questão relativa ao nível de satisfação com a disciplina, foi utilizada a escala de mensuração de Likert, rotulada: 1- Péssimo; 2- Ruim; 3Bom; 4- Muito bom, e 5- Ótimo.

Após a coleta, os dados foram armazenados em banco de dados, desenvolvido no Microsoft Office Excel, composto de planilhas independentes para cada aspecto analisado. Para a análise dos dados quantitativos foi consultado um estatístico. Os dados foram sistematizados e identificados em porcentagens de respostas, para que os resultados fossem apresentados em tabelas e discutidos a partir da literatura. A devolutiva dos dados foi realizada por meio de correio eletrônico, na qual constou uma cópia do artigo resultante da pesquisa, que foi enviado para a instituição participante, para os sujeitos entrevistados e para a Coordenação do Curso de Medicina.

\section{RESULTADOS E DISCUSSÃO}

Os dados coletados permitiram identificar o perfil dos estudantes formandos no curso de Medicina no ano de 2016, e assim analisar as percepções acerca da Medicina de Família e Comunidade para os sujeitos desta pesquisa. Vale considerar que o curso em questão foi criado em 1997 e funciona em período integral, sendo composto por 12 semestres (8 semestres básicos e préclínicos e 4 semestres de internato médico). Desde então, o passou por três mudanças curriculares e, atualmente, convive com três matrizes curriculares. As matrizes curriculares 1 e 2 têm como objetivo formar um médico generalista e policlínico, capaz de atuar na investigação, na prevenção e no tratamento das doenças, voltado especialmente para as atividades do "médico da família". $\mathrm{Na}$ matriz 3, em prática desde 2015, adequada às novas Diretrizes Curriculares Nacionais, o objetivo é formar o médico com competências relacionadas à atenção à saúde, gestão em saúde, educação permanente e continuada, de acordo com as necessidades do sistema nacional de saúde e da população brasileira.

Vale ressaltar que os sujeitos participantes deste estudo experienciaram a matriz curricular 1, sendo importante considerar novos estudos com os alunos que estão sendo formados pela matriz atual.

A análise deste artigo foi estruturada em três eixos de discussão: Caracterização do perfil dos formandos; Aspectos relacionados à MFC; e Fatores determinantes na escolha da especialidade em Medicina de Família e Comunidade.

\section{CARACTERIZAÇÃO DO PERFIL DOS FORMANDOS}

Os alunos que responderam à pesquisa apresentam distribuição da faixa etária compreendida entre 23 e 46 anos, sendo a média de idade de 26 anos. Dados semelhantes aos achados foram encontrados na literatura, dos quais se aponta um estudo que evidencia a média em anos de 25,3, variando entre 3,4 anos (SOUZA; SILVA; CALDAS, 2014), e outro que aponta a média de 23 a 24 anos (FILHO et al, 2013). Quanto ao gênero, a pesquisa identificou o predomínio do sexo feminino, totalizando $57,1 \%$ (12 sujeitos) da amostra, contra 42,8\% (9 sujeitos) do gênero masculino. Este dado se assemelha ao encontrado por Fiorotti, Rossoni e Miranda, ano de 2010, no qual houve um grande número de mulheres, com 50,2\%. Vale ressaltar que outros trabalhos também corroboram com este dado, tanto em escolas médicas do Brasil quanto no exterior. Em contrapartida, encontra-se nos estudos de Souza, Silva e Caldas (2014) e Filho et al. (2013) um dado 
divergente, no qual se evidenciou no curso médico predomínio de acadêmicos do sexo masculino, $58,3 \%$ e $54,4 \%$, respectivamente.

Quanto ao resultado encontrado, sobre o predomínio do sexo feminino na escola médica, observa-se que esta é uma tendência mundial. Vale destacar que essa mudança de ingresso das mulheres nos cursos de Medicina datam o final do último século, uma vez que em períodos anteriores elas eram consideradas com perfil inadequado para o exercer da profissão (MILLAN et al., 2005).

\section{ASPECTOS RELACIONADOS À MEDICINA DE FAMÍLIA E DA COMUNIDADE}

Este tópico apresenta os resultados encontrados a partir dos questionamentos relacionados à escolha pela área de Medicina de família e comunidade. A primeira pergunta realizada referiuse a: Já pensou em fazer residência em MFC? Assim, obteve-se como resultado que, dos sujeitos entrevistados, $57,1 \%$ responderam que já haviam pensado em fazer residência na área, conforme apresentado na Tabela 1.

Tabela 1 - Intenção dos alunos formandos em Medicina no ano de 2016/ I em realizar residência em MFC em determinado momento do curso.

\begin{tabular}{|l|c|c|}
\hline Já pensou em fazer residência em MFC em algum momento & $\mathrm{N}^{\circ}$ & $\%$ \\
\hline Sim & 12 & 57,1 \\
\hline Não & 9 & 42,9 \\
\hline
\end{tabular}

Fonte: Protocolo de pesquisa.

Neste sentido, destaca-se a dissertação de mestrado de Gomes (2011), realizada em Portugal, a qual demonstrou que a MFC estava entre as opções de escolha de 20,1\% dos ingressos, sendo que no $6^{\circ}$ ano este valor aumentou para 44,2\%. Este fato, ainda segundo a autora, revela que o contato com a MFC durante a faculdade promove uma sensibilização positiva nos estudantes em relação a esta especialidade. Considerando o processo de formação como orientador para escolha da futura área de atuação, evidencia-se o contato com disciplinas médicas durante o curso como um gerador de experiências positivas ou negativas e, consequentemente, como um instrumento auxiliar na escolha da especialidade pretendida. Assim, é relevante ressaltar a necessidade de contato do aluno com a MFC e seus subsídios, a fim de fomentar afinidade dos alunos com a especialidade (FIGUEIREDO, 2013).

Uma pesquisada realizada por Ribeiro (2006) na Faculdade de Medicina da Universidade Federal de Minas Gerais (UFMG) com 738 estudantes de vários períodos (exceto do último período) do curso apresentou como resultado que 15,4\% dos sujeitos planejavam seguir carreiras relacionadas com a atenção básica. É importante referir que nesta pesquisa foram considerados na categoria carreira na atenção básica as especialidades de clínica médica, pediatria e médico generalista. Assim, destacam-se os resultados obtidos quando considerados apenas os médicos generalistas, que apresentaram uma queda para $1 \%$.

Os alunos também foram questionados quanto ao nível de satisfação com a disciplina de MFC, os quais em sua maioria identificaram como nível 4 - muito bom (considerando o nível 1 “ péssimo" e 5 seria “ótimo”), como mostra a Tabela 2. 
Tabela 2- Nível de satisfação dos alunos formandos em Medicina no ano de 2016/ I com a disciplina de MFC.

\begin{tabular}{|l|c|c|}
\hline Nível de satisfação & $\mathrm{N}^{\mathrm{o}}$ & $\%$ \\
\hline 1 & 1 & 4,8 \\
\hline 2 & 1 & 4,8 \\
\hline 3 & 2 & 9,5 \\
\hline $\mathbf{4}$ & $\mathbf{9}$ & $\mathbf{4 2 , 9}$ \\
\hline $\mathbf{5}$ & $\mathbf{8}$ & $\mathbf{3 8 , 1}$ \\
\hline
\end{tabular}

Fonte: Protocolo de pesquisa.

Os alunos que responderam à pesquisa cursaram a matriz curricular 2 do curso. Nesta matriz, os acadêmicos tiveram seu primeiro contato com a área no $3^{\circ}$ período em uma disciplina intitulada Organização dos Serviços da Saúde e, após, no $5^{\circ}$ período, com a disciplina de Medicina Preventiva, ambas formadas por aulas teóricas. Do $5^{\circ}$ ao $8^{\circ}$ período, os alunos iniciaram a disciplina de MFC, com a divisão da turma em grupos que realizaram atividades teóricas e práticas em diferentes Unidades Básicas de Saúde, vivenciando o dia a dia do trabalho da equipe (planejamento local, territorialização, atenção domiciliar, grupos de apoio, entre outros.). No internato, do $9^{\circ}$ ao $11^{\circ}$ período, os alunos obtiveram mais autonomia no quesito clínico, ao experienciar as consultas, papel também desempenhado na prática do médico de família. Nóbrega-Therrien et al. (2015), em pesquisa realizada com residentes em MFC no Ceará, inferiu que novos currículos com mais disciplinas e estágios voltados para a Atenção Primária/Saúde Comunitária/Medicina de Família permitem que os médicos recém-formados se sintam mais preparados para atuar na ESF. Em outra universidade, na Universidade Cidade de São Paulo, os alunos encaram a MFC tanto como uma fonte de motivação (que os possibilitou conhecer realidades diferentes, situações novas e ter contato com diferentes realidades) quanto uma obrigação curricular, baseada em repetição de atividades e cansaço (RESTOM, 2015).

Alguns motivos para o fraco desejo por seguir a área de MFC foram apontados em teses de mestrado, como a "Experiência em MFC com um profissional desmotivado e frustrado", a "Pouca vivência em MFC durante o curso" e também "Estágio pouco interessante" (GOMES, 2011). Um estudo do ano de 2003 revela que as faculdades que têm um programa longitudinal, que mantêm o aluno em contato com a MFC, parecem influenciar o número de estudantes que escolhe esta especialidade (SENF; CAMPOS-OUTCALT; KUTOB, 2003).

\section{ASPECTOS ENVOLVIDOS NA ESCOLHA DA ESPECIALIDADE EM MEDICINA DE FAMÍLIA E COMUNIDADE}

A fim de identificar os motivos que levariam os alunos a fazer residência em MFC, o questionário aplicado continha tópicos a serem assinalados (poderia assinalar mais de uma opção), além de que os alunos poderiam citar algum outro motivo caso este não estivesse listado dentre as opções. Na Tabela 3 é possível identificar os motivos favorecedores, entre os entrevistados, pela opção por MFC como especialidade médica.

Infere-se que os fatores mais levados em conta na opinião dos acadêmicos foram em primeiro lugar a "carga horária relativamente baixa com horários definidos", juntamente com "olhar médico ampliado, além do biomédico”, ambos correspondendo a 57,1\% (12 sujeitos), seguido 
da "oportunidade de trabalho em qualquer região", 52,4\% (11 sujeitos) e por fim a "atuação ampla em diversas disciplinas médicas”, 47,6\% (10 sujeitos).

Tabela 3- Motivos pelos quais os formandos em Medicina no ano de 2016/ I se sentem atraídos pela MFC.

\begin{tabular}{|l|c|c|}
\hline Motivos pelos quais se sentem atraídos & $\mathbf{N}^{\mathbf{0}}$ & $\%$ \\
\hline Olhar médico ampliado, além do biomédico & 12 & 57,1 \\
\hline Carga horária relativamente baixa com horários definidos (flexibilidade) & 12 & 57,1 \\
\hline Oportunidade de trabalho em qualquer região & 11 & 52,4 \\
\hline $\begin{array}{l}\text { Atuação ampla em diversas disciplinas médicas } \\
\text { Alta resolutividade do grupos }\end{array}$ & 3 & 47,6 \\
\hline $\begin{array}{l}\text { Atendimento além do consultório (visitas domiciliares, } \\
\text { comunitários) }\end{array}$ & 2 & 19,0 \\
\hline Outros *
\end{tabular}

Fonte: Protocolo de pesquisa.

* Os outros itens citados foram: Carga horária mais flexível em relação à GO (1 sujeito), financeiro relativamente bom (1 sujeito).

Dorsey, Jarjoura e Rutecki (2003) já haviam demonstrado que um fator determinante na escolha profissional de $55 \%$ dos estudantes de escolas médicas norte-americanas é um estilo de vida na qual se pode ter controle. A "Disponibilidade para vida familiar" (83\%) foi um motivo bastante valorizado pelos estudantes na tese de Gomes (2011). Neste estudo, "Especialidade que abrange uma grande variedade de patologias $(76 \%)$ e "Possibilidade de atuar na prevenção da doença e na promoção de saúde e de continuidade de cuidados” (71\%) também foram motivos bastante citados. Segundo o Ministério da Saúde, a atenção primária fornece serviços de saúde pública em diversas disciplinas médicas, incluindo saúde da criança, saúde da mulher, saúde do idoso, saúde bucal, além de questões mais específicas, tais como saúde do hipertenso e do diabético, como também ações de promoção da saúde. Envolve também a vigilância epidemiológica e sanitária de sua área adstrita, garantindo uma redução das desigualdades sociais e regionais (BRASIL, 2007). Além disso, a Atenção Primária deve considerar o sujeito em sua singularidade, complexidade, integralidade e inserção no meio, ou seja, o olhar sobre o indivíduo deve ser ampliado, além do biomédico (BRASIL, 2004).

Segundo Anderson, Gusso e Filho (2005), cerca de 85\% dos problemas de saúde relativos a uma população específica, sem diferenciação de sexo ou faixa etária, podem ser resolvidos por uma equipe de estratégia de saúde da família, na qual se inclui o médico de família. Não existe consenso sobre qual o número ideal de população que o médico de família deva atender, mas se estima que entre 1.800 e 2.200 pessoas lhe permitiria manter sua resolubilidade e disponibilidade (GUSSO; LOPES, 2012).

Da mesma forma, os alunos responderam o questionário com perguntas, a fim de identificar os motivos pelos quais eles não fariam residência em MFC, conforme exposto na Tabela 4.

Pode-se observar que o motivo mais apontado foi "más condições de trabalho e o pouco investimento por parte do governo”, correspondendo a 76,2\%, citado por 16 sujeitos. Entre os fatores que levam à não atração dos profissionais de saúde para o trabalho na ESF, estão as condições inadequadas de trabalho, baixos salários, equipes de trabalho incompletas, demanda excessiva e falta de estímulo ao crescimento profissional (NÓBREGA-THERRIEN, 2015). Insatisfação no trabalho em saúde tem implicações na saúde dos profissionais e nos resultados da assistência 
prestada por ele (MELO; BARBOSA; SOUZA, 2011).

Tabela 4- Motivos pelos quais os formandos em Medicina no ano de 2016/ I não se sentem atraídos pela MFC.

\begin{tabular}{|l|c|c|}
\hline Motivos pelos quais não se sentem atraídos & $\mathrm{N}^{\circ}$ & $\%$ \\
\hline Más condições de trabalho/pouco investimento pelo governo & 16 & 76,2 \\
\hline Baixa remuneração & 11 & 52,4 \\
\hline Pouco prestígio profissional (baixa valorização) & 6 & 28,6 \\
\hline Experiências com profissionais desmotivados/frustrados & 6 & 28,6 \\
\hline Ter que trabalhar no SUS & 3 & 14,3 \\
\hline Sensação de que MFC “é fácil de mais” & 2 & 9,5 \\
\hline Outros*: & 2 & 9,5 \\
\hline Necessidade de acompanhamento longitudinal do paciente & 1 & 4,8 \\
\hline Pouca vivência na área & 0 & 0,0 \\
\hline
\end{tabular}

Fonte: Protocolo de pesquisa.

* Os itens citados foram: Gosto pelo trabalho intra-hospitalar (1 sujeito) e Baixa resolutividade (1 sujeito).

Para Oliveira Junior (2013), a escassez de profissionais atuando na atenção primária, associada à falta de equipamentos, materiais e medicamentos, acaba inviabilizando as práticas de trabalho. Situações de sofrimento na vivência cotidiana dos diferentes profissionais incluem materiais e equipamentos obsoletos e/ou em condições precárias de manutenção, limitações de espaço físico e limitação de profissionais frente à demanda.

Em segundo lugar, a "baixa remuneração” foi um fator citado por vários acadêmicos, sendo o motivo mais recorrente para desmotivação em seguir a carreira em MFC de acordo com Neto, Lira e Miranda (2009), realizada a partir da revisão de diversos trabalhos já publicados. Segundo estes autores, o médico de família até recebe boa remuneração atuando na ESF, porém o salário final deste profissional ainda é inferior ao de outros especialistas.

Outro aspecto bastante citado é a visão de "pouco prestígio profissional” na área da MFC. Essa visão de que o médico generalista possui um menor valor desperta desconfiança e se traduz no baixo interesse dos egressos em realizar residência em (MAGALHÃES; BELMONTE; LUNA, 2014). A Atenção Básica é vista como um setor de baixa complexidade médica, composta por profissionais mal treinados e desestimulados na profissão, julgados como médicos de "segunda classe" (MELLO et al., 2009).

Muito dos motivos citados pelos alunos foram apontados também por Nóbrega-Therrien et al. (2015) em estudo feito com residentes que tiveram contato com a ESF durante a graduação e após formados, sendo as dificuldades apontadas por eles as condições inadequadas de trabalho, os baixos salários, a falta de estímulo ao crescimento profissional e, junto a isso, a falta de reconhecimento desse profissional. Alguns obstáculos precisam ser superados, dentre eles a pouca valorização da carreira, qualificação profissional insuficiente, baixa remuneração, pouco prestígio social e deficiência de programas de pós-graduação (NETO; LIRA; MIRANDA, 2009). Um outro estudo feito por Gaspar (2006) aponta para a relevância do papel dos professores como modelos, permitindo um efeito positivo nas escolhas dos estudantes por carreiras generalistas.

Por fim, no questionário aplicado havia uma questão referente à escolha pela residência após a formatura. Como resultado, obteve-se como escolha a residência em Clínica Médica, correspondendo à escolha de 7 sujeitos (33,3\%), seguido de Clínica Cirúrgica (14,3\%). Oftalmologia, 
Ginecologia e Obstetrícia, Otorrinolaringologia, Medicina Intensiva e MFC foram citadas, cada uma por 2 alunos (9,5\%), além de Pediatria, Emergência, Ortopedia, Anestesiologia e Radiologia, citadas por um aluno cada $(4,8 \%)$, conforme observado na Tabela 5 .

Tabela 5- Residência médica que os formandos do curso de medicina no ano de 2016/I tem interesse em fazer.

\begin{tabular}{|l|c|c|}
\hline Residência que pretende fazer após formado & $\mathrm{N}^{\circ}$ & $\%$ \\
\hline Clínica Médica & 7 & 33,3 \\
\hline Cirurgia Geral & 3 & 14,3 \\
\hline Oftalmologia & 2 & 9,5 \\
\hline Ginecologia e Obstetrícia & 2 & 9,5 \\
\hline MFC & 2 & 9,5 \\
\hline Otorrinolaringologia & 2 & 9,5 \\
\hline Medicina Intensiva & 2 & 9,5 \\
\hline Pediatria & 1 & 4,8 \\
\hline Emergência & 1 & 4,8 \\
\hline Ortopedia & 1 & 4,8 \\
\hline Anestesiologia & 1 & 4,8 \\
\hline Radiologia & 1 & 4,8 \\
\hline
\end{tabular}

Fonte: Protocolo de Pesquisa.

Este dado contrapõe o estudo de Sousa, Silva e Caldas (2014), no qual houve predomínio de interesse pela residência em Oftalmologia (14\%), seguida por Pediatria (12,3\%) e Otorrinolaringologia e Radiologia (ambos 8,8\%), não chegando a citar a área de Clínica Médica. Já Belarmino, Martins e Franco (2016) obtiveram em seu estudo a predominância de escolha por Cirurgia Geral (11,4\%), Pediatria (7,5\%) e Oftalmologia (7,1\%). Este mesmo trabalho, a partir da análise de outros estudos, afirma que especialidades como Medicina da Família, Pediatria, Cirurgia Geral, Ginecologia e Obstetrícia são cada vez menos requisitadas pelos estudantes de Medicina.

Oliveira e Alves (2011), em pesquisa na Universidade do Pará, viram que 64\% dos alunos relataram desejo de ser "especialistas", dos quais $41 \%$ gostariam de atuar na clínica e 23\% na cirurgia. Neste mesmo estudo, somente 20\% declararam que gostariam e começariam a trabalhar em Medicina Geral, como o Programa Saúde da Família, contradizendo outro dado, em que 65\% afirmaram que se sentiam mais seguros para atender "casos clínicos em geral”.

\section{CONSIDERAÇÕES FINAIS}

Considerando o objetivo deste estudo, obteve-se que o perfil dos formandos é semelhante ao encontrado em outros trabalhos, sendo importante na formulação da graduação e suas respectivas mudanças. Os resultados limitaram-se ao número de amostra que se conseguiu obter no momento da pesquisa.

O perfil do egresso de Medicina é de jovens com idade média de 26 anos, em sua maioria mulheres, que dizem já ter pensado em fazer residência médica em MFC. Os formandos dizem-se satisfeitos com a disciplina ministrada na instituição investigada, atribuindo uma nota 4 (muito bom) na avaliação. Por fim, os alunos sentem-se atraídos pela MFC devido à "carga horária relativamente baixa com horários definidos", ao "olhar médico ampliado, além do biomédico”, além do fato de a MFC possibilitar a "oportunidade de trabalho em qualquer região" e a "atuação 
ampla em diversas disciplinas médicas". Já os motivos que não os atraem são as "más condições de trabalho e o pouco investimento por parte do governo", a "baixa remuneração" e o "pouco prestígio profissional" na área.

Com isso, é possível concluir que o contato com a MFC durante a graduação é um ponto favorecedor para a formação de profissionais interessados na área. Soma-se a isso o estilo de vida que o médico de família consegue ter. Uma questão a se refletir e discutir é a falta de investimento pelo governo nas condições de trabalho dos profissionais, tanto no que se refere ao investimento financeiro e salarial, quanto em relação ao prestígio que esse profissional merece.

Diante do exposto, é preciso ressaltar a relevância de se entender qual o sentimento que a MFC desperta nos acadêmicos de Medicina. No presente estudo, é possível observar que se disserem satisfeitos com a disciplina ministrada e apenas 2 alunos (9,5\%) citaram que a MFC seria uma escolha possível de residência. A maioria dos alunos já pensou pelo menos uma vez em atuar na área de MFC, o que corresponde apenas à percepção dos mesmos sobre a área, e não a possibilidade real de escolha pela especialidade.

A não escolha por esta área pode estar relacionada aos motivos citados pelos sujeitos desta pesquisa, no que se refere à baixa remuneração, ao pouco investimento pelo governo e à baixa valorização da especialidade. Por isso, deve-se atentar ao fato de que possivelmente a escolha final esteja atrelada ao mercado de trabalho a que o médico recém-formado se depara. Muitos médicos acabam trabalhando na Atenção Primária temporariamente, simplesmente por não ter conseguido entrar na residência de seu interesse. Outros trabalham na ESF por ela não exigir uma residência prévia e depois escolhem outra área que lhe seja mais conveniente. Além do mais, o fato de a maioria dos lugares não possuírem um plano de carreira, e o salário de um médico de família ainda ser inferior comparado às demais especialidades médicas, desmotivam o profissional a trabalhar exclusivamente na área.

Mesmo frente ao movimento de mudança desta realidade proposta pelo governo por meio de programas e políticas que induzem à formação médica, observa-se que estas ainda são incipientes, e assim continuarão se não propuserem uma maior consolidação e incentivo financeiro para valorização profissional desta área.

\section{REFERÊNCIAS}

ANDERSON, M. I. P.; DEMARZO, M. M. P.; RODRIGUES, R. D. A medicina de família e comunidade, a Atenção Primária à Saúde e o Ensino de Graduação: recomendações e potencialidades. Revista Brasileira de Medicina de Família e Comunidade, v. 3, n. 11, p. 157-172, 2007.

ANDERSON, M. I. P.; GUSSO, G.; FILHO, E. D. C. Medicina de Família e Comunidade: especialistas em integralidade. Revista de APS, v. 8, n. 1, p. 61-67, jan/jun, 2005.

BELARMinO, L. N. M.; MARTINS, M. F.; FRANCO, M. C. A. Aspirações Médicas: Análise dos Alunos do Internato das Instituições de Ensino Superior do Estado do Pará. Revista Brasileira de Educação Médica, vol. 40, n. 4, p. 685-693, 2016.

BRASIL. Conselho Nacional de Secretários de Saúde. Atenção Primária- Seminário do Conass para construção de consensos / Conselho Nacional de Secretários de Saúde. - Brasília: CONASS. 44 p., (CONASS Documenta; v.2), 2004.

BRASIL. Ministério da Saúde. Secretaria de Atenção À Saúde. Departamento de Atenção Básica. Política 
Nacional de Atenção Básica / Ministério da Saúde, Secretaria de Atenção à Saúde, Departamento de Atenção Básica. - 4. ed. - Brasília: Ministério da Saúde. 68 p. - (Série E. Legislação de Saúde) (Série Pactos pela Saúde 2006; v. 4). 2007.

CAMPOS, C. E. A. Os princípios da medicina de família e comunidade. Revista de APS, v. 8, n. 2, p. 181190, jul/dez, 2005.

CARDOSO FILHO, F. A. et al. Perfil do Estudante de Medicina da Universidade do Estado do Rio Grande do Norte (UERN) em 2013. Revista Brasileira de Educação Médica, v. 39, n. 1, p. 32-40, 2015.

DORSEY, E. R.; JARJOURA, D.; RUTECKI G. W. Influence of controllable lifestyle on recent trends in specialty choice by US medical students. JAMA. v. 290, n. 9, p.1173-8, 2003.

FIGUEIREDO, P. H. M. Escolha da especialidade em medicina de família e comunidade entre alunos concluintes dos módulos do internato em medicina de família e comunidade na Universidade Federal do Pará. São Paulo. Mestrado [dissertação] - Universidade Federal de São Paulo, 2013.

FIOROTTI, K. P.; ROSSONI, R. R.; MIRANDA, A. E. Perfil do Estudante de Medicina da Universidade Federal do Espírito Santo, 2007. Revista Brasileira de Educação Médica, v. 34, n. 3, p. 355-362, 2010.

GASPAR, D. Medicina Geral e Familiar: uma escolha gratificante. Acta Méd. Port, v. 19, p. 133-9, 2006.

GOMES, A. R. C. Medicina Geral e Familiar: Do ensino à escolha da especialidade. Covilhã. Mestrado [dissertação] - Universidade da Beira Interior, 2011.

GUSSO, G.; LOPES, J. M. C. Tratado de Medicina de Família e Comunidade: Princípios, formação e prática (Volume II). Porto Alegre: Artmed, 2012.

JANAUDIS, M. A. et al. Formando médicos para a Medicina de Família e Comunidade. Revista Bioética. v. 15, n. 1 , p. $27-36,2007$.

LOPES, J. M. C.; RIBEIRO, J. A. R. A pessoa como centro do cuidado na prática do médico de família. Revista Brasileira de Medicina de Família e Comunidade, v.10, n. 34, p. 1-13, 2015.

LUCCHESE, P. T. R. Equidade na gestão descentralizada do SUS: desafios para a redução de desigualdades em saúde. Ciência \& Saúde Coletiva, v. 8, n. 2, p. 439-448, 2003.

MAGAlhães, T. N.; BELMONTE, T. S. A.; LUNA, C. A. A Medicina de Família na Educação Médica: Um Núcleo de Ensino na Atenção Terciária para a Aprendizagem em Atenção Primária. Cadernos Brasileiro de Medicina, v. 27, n. 3, p. 1-58, 2014.

MELlo, G. A. et al. Médico de família: ser ou não ser? Dilemas envolvidos na escolha desta carreira. Revista Brasileira de Educação Médica, v. 33, n. 3, p. 475 - 482, 2009.

MELO, M. B.; BARBOSA, M. A.; SOUZA, P. R. Satisfação no trabalho da equipe de enfermagem: revisão integrativa. Revista latino-americana de Enfermagem; v. 19, n. 4, p.1047-55, 2011.

MILLAN, L. R. et al. What is behind a student's choice for becoming a doctor? Clinics, v. 60, n. 2, p. 143$50,2005$.

NETO, P. G. C.; LIRA, G. V.; MIRANDA, A. S. Interesse dos Estudantes pela Medicina de Família: Estado da Questão e Agenda de Pesquisa. Revista Brasileira de Educação Médica, v. 33, n. 2, p. 198204; 2009.

NÓBREGA-THERRIEN, S. M. et al. Formação para a Estratégia Saúde da Família na Graduação em 
Medicina. Revista Brasileira de Educação Médica, v. 39, n. 1, p. 112-118; 2015.

RBTS, v.4, n.1, $2017-87$

OLIVEIRA JUNIOR; R. G. Condições de trabalho das Equipes de Saúde da Família do município de Petrolina-PE: percepção dos profissionais de saúde. O Mundo da Saúde; v. 37, n.4, p. 433-438, 2013.

OLIVEIRA, N. A.; ALVES, L. A. Ensino Médico, SUS e início da profissão: como se sente quem está se formando? Revista Brasileira de Educação Médica; v. 35, n. 1, p. 26-36, 2011.

RESTOM, A. G. Representação Social das Vivências de Estudantes no Curso de Medicina. Revista Brasileira de Educação Médica, v. 39, n. 3, p. 370-377, 2015.

RIBEIRA, M. M. F. Avaliação da atitude do estudante de medicina da Universidade Federal de Minas Gerais, a respeito da relação médico-paciente, no decorrer do curso médico. Doutorado [tese] - Universidade Federal de Minas Gerais, 2006.

RODRIGUES, R. D.; ANDERSON, M. I. P. Saúde da Família: uma estratégia necessária. Revista Brasileira de Medicina de Família e Comunidade, v. 6, n. 18, p. 21-24, 2011.

SENF, J. H.; CAMPOS-OUTCALT, D.; KUTOB, R. Factors related to the choise of Family Medicine: A Reassessment and Literature Review. JABFP; v. 16, n. 6, p. 502-12, 2003.

SILVA, N. R. Fatores determinantes da carga de trabalho em uma unidade básica de saúde. Ciência \& Saúde Coletiva, v. 16, n. 3, p. 3393-3402, 2011.

SOUSA, I. Q.; SILVA, C. P.; CALDAS, C. A. M. Especialidade médica: escolhas e influências. Revista Brasileira de Educação Médica, v. 38, n. 1, p. 79-86, 2014. 